\title{
Quadriplegia after Mitral Valve Replacement in an Infective Endocarditis Patient with Cervical Spine Spondylitis
}

\author{
Ji Min Lee, M.D., Seon Yeong Heo, M.D., Dong Kyu Kim, M.D., Jong Pil Jung, M.D., Chang Ryul Park, M.D., \\ Yong Jik Lee, M.D., Gwan Sic Kim, M.D. \\ Department of Thoracic and Cardiovascular Surgery, Ulsan University Hospital, University of Ulsan College of Medicine, Ulsan, Korea
}

\section{ARTICLE INFO}

Received May 20, 2020

Revised August 28, 2020

Accepted September 1, 2020

\section{Corresponding author}

Gwan Sic Kim

Tel 82-52-250-8726

Fax 82-52-250-8558

E-mail 0734519@uuh.ulsan.kr

ORCID

https://orcid.org/0000-0002-0648-0178

\begin{abstract}
The simultaneous incidence of infective endocarditis and cervical spondylitis with an epidural abscess is rare, and quadriplegia as a complication after cardiac surgery is very rare. We recently observed quadriplegia after mitral valve replacement in an infective endocarditis patient with cervical spine spondylitis. With early symptom detection, immediate examination, and prompt surgical treatment, the patient successfully recovered without neurological symptoms.
\end{abstract}

Keywords: Mitral valve replacement, Quadriplegia, Spondylitis

\section{Case report}

A 50-year-old man was admitted to the neurosurgery department via the emergency room with neck pain and general weakness that had lasted several days. The patient's medical history included smoking and chronic hepatitis B. He denied any history of trauma or recent invasive procedures. His vital signs were as follows: blood pressure, 122/85 mm Hg; regular heart rate, 85 beats/min; respiratory rate, 20 cycles $/ \mathrm{min}$; and body temperature, $39^{\circ} \mathrm{C}$. A neurological examination by a neurosurgeon yielded normal results. Laboratory tests revealed a white blood cell count of $10,190 / \mathrm{mm}^{3}$, aspartate aminotransferase and alanine aminotransferase levels of $114 \mathrm{IU} / \mathrm{L}$ and $90 \mathrm{IU} / \mathrm{L}$, respectively, and C-reactive protein levels of $1.21 \mathrm{mg} / \mathrm{dL}$; other parameters were within normal limits. Initial chest radiography and a 12-lead electrocardiogram were unremarkable. Magnetic resonance imaging (MRI) of the cervical spine revealed an abscess with inflammatory changes in the epidural space and prevertebral portion at the C4-C5 level, suggesting infectious spondylitis (Fig. 1A). Subsequent transthoracic echocardiography (TTE) and transesophageal echocardiography (TEE) identified a 1.3-cm hyper-mobile vegetation on the anterior mitral valve leaflet with severe mitral regurgitation. The size and systolic function of the
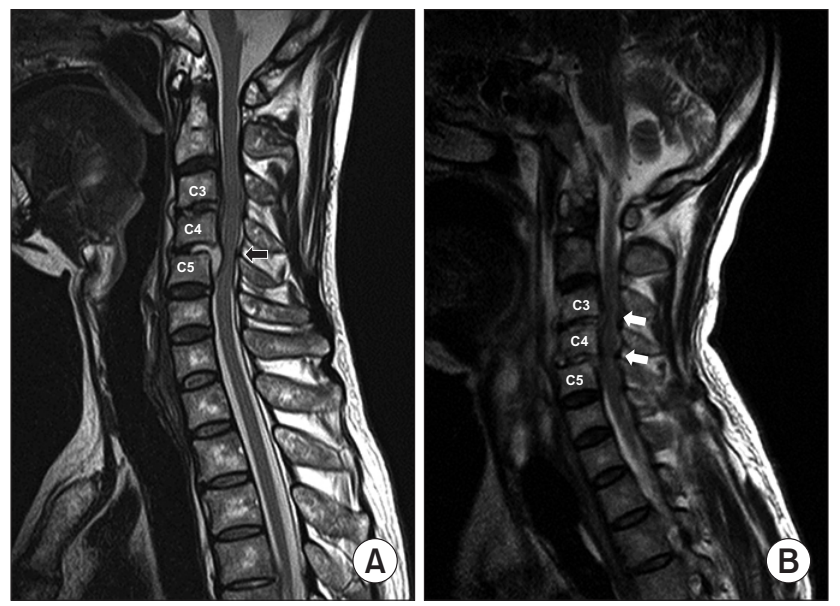

Fig. 1. (A) Initial cervical MRI showing an abscess with inflammatory changes in the epidural space and prevertebral portion of C4-C5 (black arrow). (B) After cardiac surgery, emergent wholespine MRI revealed progression of the epidural abscess and a compressed spinal cord at C3-C5 (white arrows). MRI, magnetic resonance imaging.

left and right ventricles were normal. Because of the systemic embolic risk posed by the hyper-mobile intracardiac mass, the patient was referred to the department of thoracic and cardiovascular surgery at Ulsan University Hospital for surgical treatment. Emergent surgery was undertaken. The 
operation was performed using ascending aorta and right atrium cannulation under moderate hypothermia. Antegrade cold blood cardioplegia was used for myocardial protection. Operative findings showed a vegetation on the anterior mitral valve leaflet. Mitral valve replacement (St. Jude Medical Regent 27 mm; St. Jude Medical Inc., St. Paul, MN, USA) was performed without complications. After cardiac surgery, the patient was transferred to the cardiac intensive care unit (ICU) while intubated and sedated. After admission to the ICU, his vital signs were normal and urine output was satisfactory. Approximately 6 hours after the operation, he opened eyes spontaneously and nodded according to instructions. However, he was unable to move his extremities. A detailed neurological examination showed flaccid paralysis of all 4 limbs, with loss of sensation below the T8 dermatome level. The upper and lower limb muscle strengths were 1/5 and 0/5, respectively. Emergent wholespine MRI revealed progression of an epidural abscess and a compressed spinal cord at C3-C5 (Fig. 1B). Immediately, a neurosurgical consultation was conducted followed by corpectomy and abscess removal (12 hours after heart surgery) using an anterior approach via the right side of the neck. The patient returned to the ICU while intubated and sedated. He regained consciousness, mobility, and feeling in his extremities 3 hours after surgery. On postoperative day 1 , he was weaned and extubated; muscle strength was restored to all 4 limbs (grade 5/5). On postoperative day 4 , he was transferred to the general ward. TTE on postoperative day 6 showed a well-positioned, functional prosthetic valve without paravalvular leak or dehiscence. On postoperative day 9, cervical-spine MRI showed successful abscess removal and a decompressed spinal cord at C3-C5 (Fig. 2). The patient was able to walk around the ward with a neck brace. Preoperative blood culture indicated the presence of Streptococcus agalactiae; however, intraoperative tissue cultures from both cardiac and spine surgery were negative for it. Preoperatively, empirical antibiotics (meropenem [1 g every 8 hours] and vancomycin [ $1 \mathrm{~g}$ every 12 hours]) were administered for 3 days. Subsequently, according to the sensitivity test for antibiotics, the preoperative antibiotics (ampicillin [2 g every 4 hours] and gentamicin $[1 \mathrm{mg} / \mathrm{kg}$ every 8 hours]) were changed and administered for 5 days. Postoperatively, intravenous antibiotics (ampicillin $[2 \mathrm{~g}$ every 4 hours] and gentamicin [1 mg/kg every 8 hours]) were administered for 4 weeks. On postoperative day 29, the patient was discharged without neurological abnormalities. At a 12-month follow-up, the patient's postoperative course was uneventful and clinical findings were normal without any neurological symptoms. The patient provided written

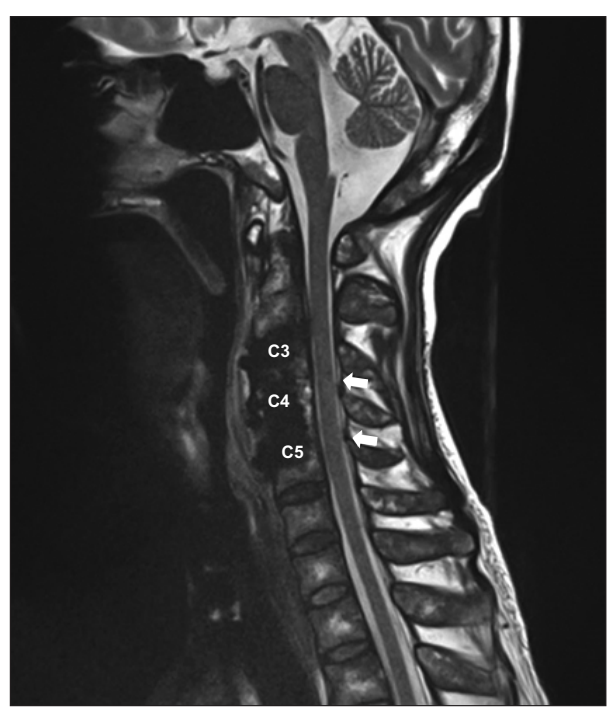

Fig. 2. After emergent neurosurgery, follow-up magnetic resonance imaging of the cervical spine showed successful removal of the abscess and a decompressed spinal cord at C3-C5 (white arrows).

informed consent for publication of his clinical details and images.

\section{Discussion}

The simultaneous occurrence of infective endocarditis and cervical epidural abscess is rare [1], and quadriplegia as a complication after cardiac surgery is very rare and poorly understood [2-6]. The lack of management and treatment guidelines for this complication pose significant challenges for cardiac surgeons. Here, we report a case of quadriplegia after mitral valve replacement in an infective endocarditis patient with cervical spine spondylitis.

Spinal epidural abscess shows an incidence of 0.2-1.2 patients per 10,000 inpatients. A MEDLINE search of infective endocarditis showed an incidence of vertebral osteomyelitis of $6.5 \%$ among 1,099 patients with infective endocarditis [1]. In particular, the occurrence of cervical spine abscess accompanied by infective endocarditis is rarer than the occurrence of abscesses in the lumbar and thoracic spine.

Spinal epidural abscess without neurological deficits can be safely and efficiently managed with conservative treatment $[7,8]$. Therefore, if infective endocarditis and cervical epidural abscess occur at the same time, as in this case, cardiac surgery can be performed alone if the lesion of infective endocarditis needs an emergent operation. However, insufficient data have been published on the deteriora- 
tion of spinal epidural abscesses and the occurrence of neurological complications after cardiac surgery.

There are several potential causes of quadriplegia after cardiac surgery in infective endocarditis patients with a cervical epidural abscess. First, cardiopulmonary bypass $(\mathrm{CPB})$ induces an acute-phase reaction with the activation of leukocytes and endothelial cells. Therefore, CPB can exacerbate systemic inflammatory processes and contribute to epidural abscess progression. Second, the insufficient use of antibiotics in an emergency situation may also cause this outcome. Third, during conventional cardiac surgery, physical positioning may cause neck hyperextension, which can promote cord injury if prolonged. Other procedures such as tracheal intubation and TEE, which is performed during cardiac surgery, may also promote neck hyperextension. Therefore, it is necessary to consult with the surgical team and anesthesiologist in advance and closely monitor neck hyperextension. To minimize the risk of neck hyperextension, during surgery, the head and neck should be maintained in a neutral position along the coronal plane by placing either pillows or gel pads under the patient's head. However, we overlooked the patient's cervical spine lesion and believe that neck hyperextension significantly contributed to the exacerbation of the lesion. Additionally, $S$. agalactiae (group B streptococcus) is as virulent as Staphylococcus aureus [9]. Therefore, the strain itself is also thought to have affected the exacerbation of the disease.

In regard to the management of spinal cord compression by an epidural abscess, early symptom detection, immediate examination, and prompt surgical decompression are important for a good prognosis. In particular, limiting the time from symptom onset to surgical treatment improves the prognosis. Heusner [10] reported that a complete recovery is possible if preoperative paresis does not last for longer than 36 hours, favorable if it lasts for 36-48 hours, and unfavorable if it lasts for 48 hours or longer. We detected our patient's symptoms 6 hours following cardiac surgery and followed up with an immediate examination. Twelve hours following cardiac surgery, emergent decompression surgery was performed. It is believed that the rapid management from symptom onset to decompression surgery contributed to the patient's complete recovery.

We describe a unique case of quadriplegia after mitral valve replacement in an infective endocarditis patient with cervical spine spondylitis. The quadriplegia was caused by an epidural abscess and spinal cord compression at the C3-C5 level. With early symptom detection, immediate examination, and prompt surgical treatment, the patient recovered without neurological symptoms. When a patient with cervical spinal spondylitis undergoes cardiac surgery, the possibility of cervical spinal cord lesion exacerbation should be considered.

\section{Conflict of interest}

No potential conflict of interest relevant to this article was reported.

\section{ORCID}

Ji Min Lee: https://orcid.org/0000-0001-9317-0313

Seon Yeong Heo: https://orcid.org/0000-0003-4857-7100

Dong Kyu Kim: https://orcid.org/0000-0002-0047-2732

Jong Pil Jung: https://orcid.org/0000-0002-2992-7729

Chang Ryul Park: https://orcid.org/0000-0003-2841-7046

Yong Jik Lee: https://orcid.org/0000-0002-0837-4336

Gwan Sic Kim: https://orcid.org/0000-0002-0648-0178

\section{References}

1. Oh JS, Shim JJ, Lee KS, Doh JW. Cervical epidural abscess: rare complication of bacterial endocarditis with Streptococcus viridans: a case report. Korean J Spine 2015;12:22-5.

2. Hwang NC, Singh P, Chua YL. Quadriparesis after cardiac surgery. J Cardiothorac Vasc Anesth 2008;22:587-9.

3. Samiotis I, Baikoussis NG, Patris V, Argiriou M, Dedeilias P, Charitos C. Coronary artery bypass grafting and paraparesis; is there a correlation? Cardiovasc J Afr 2018;29:e6-8.

4. Tan AK, Naik MJ, Chua YL. Quadriplegia post-coronary artery bypass graft surgery: an unusual case. Asian Cardiovasc Thorac Ann 2018;26:390-1.

5. Fujioka S, Niimi Y, Hirata K, Nakamura I, Morita S. Tetraplegia after coronary artery bypass grafting. Anesth Analg 2003;97:979-80.

6. Baek WK, Kim YS, Kim JT, Yoon BN. Critical illness neuromyopathy complicating cardiac surgery. Acute Crit Care 2018;33:51-6.

7. Siddiq F, Chowfin A, Tight R, Sahmoun AE, Smego RA Jr. Medical vs surgical management of spinal epidural abscess. Arch Intern Med 2004;164:2409-12.

8. Sorensen P. Spinal epidural abscesses: conservative treatment for selected subgroups of patients. Br J Neurosurg 2003;17:513-8.

9. Sambola A, Miro JM, Tornos MP, et al. Streptococcus agalactiae infective endocarditis: analysis of 30 cases and review of the literature, 1962-1998. Clin Infect Dis 2002;34:1576-84.

10. Heusner AP. Nontuberculous spinal epidural infections. N Engl J Med 1948;239:845-54. 